\title{
BMJ Open Cohort profile: the Finnish Genetics of Pre-eclampsia Consortium (FINNPEC)
}

\author{
Tiina Jääskeläinen, ${ }^{1}$ Seppo Heinonen, ${ }^{2}$ Eero Kajantie, ${ }^{3,4,5}$ Juha Kere,,${ }^{6,7,8}$ \\ Katja Kivinen, ${ }^{9}$ Anneli Pouta, ${ }^{5,10}$ Hannele Laivuori, ${ }^{1,2,11}$ for the FINNPEC \\ Study Group
}

To cite: Jääskeläinen $T$, Heinonen S, Kajantie E, et al. Cohort profile: the Finnish Genetics of Pre-eclampsia Consortium (FINNPEC). BMJ Open 2016;6:e013148. doi:10.1136/bmjopen-2016013148

- Prepublication history for this paper is available online. To view these files please visit the journal online (http://dx.doi.org/10.1136/ bmjopen-2016-013148).

Received 23 June 2016 Revised 12 October 2016 Accepted 18 October 2016

CrossMark

For numbered affiliations see end of article.

\section{Correspondence to} Dr Tiina Jääskeläinen; tiina.j.jaaskelainen@helsinki.fi

\section{ABSTRACT}

Purpose: The Finnish Genetics of Pre-eclampsia Consortium (FINNPEC) Study was established to set up a nationwide clinical and DNA database on women with and without pre-eclampsia (PE), including their partners and infants, in order to identify genetic risk factors for PE.

Participants: FINNPEC is a cross-sectional casecontrol cohort collected from 5 university hospitals in Finland during 2008-2011. A total of 1450 patients with PE and 1065 pregnant control women without PE (aged 18-47 years) were recruited. Altogether, there were 1377 full triads (625 PE and 752 control triads).

Findings to date: The established cohort holds both clinical and genetic information of mother-infant-father triads representing a valuable resource for studying the pathogenesis of the disease. Furthermore, maternal biological samples (first and third trimester serum and placenta) will provide additional information for $\mathrm{PE}$ research. Until now, research has encompassed studies on candidate genes, Sanger and next-generation sequencing, and various studies on the placenta. FINNPEC has also participated in the InterPregGen study, which is the largest investigation on maternal and fetal genetic factors underlying PE until now.

Future plans: Ongoing studies focus on elucidating the role of immunogenetic and metabolic factors in PE. Data on morbidity and mortality will be collected from mothers and fathers through links to the nationwide health registers.

\section{INTRODUCTION}

Pre-eclampsia (PE) is a complex vascular pregnancy disorder. PE begins with a placental trigger followed by a maternal systemic response. The condition is heterogeneous in origin, which results in considerable variation in the clinical presentation. ${ }^{1}$ Its classical clinical features are maternal hypertension and proteinuria developing after the 20th week of gestation. The rest of the systemic features can vary from mild cases with little systemic involvement to multiorgan failure. ${ }^{2}$ This has often led to inconsistent results in attempts to understand PE on the basis of a single pathogenetic hypothesis.

\section{Strengths and limitations of this study}

- The established cohort holds both clinical and genetic information of mother-infant-father triads representing a resource for studying the pathogenesis of pre-eclampsia (PE).

- Maternal biological samples (first and third trimester serum and placenta) provide a possibility for further PE research.

- A major strength of this study is its large multicentre prospective design with opportunities for follow-up through linking to the national data sources as well as the recalling of participants for future studies.

- The Finnish Genetics of Pre-eclampsia Consortium (FINNPEC) cohort also includes valuable information on socioeconomic position such as educational attainment and occupational status.

- Women with PE in this cohort have been referred to university hospitals, and thus may represent the more severe end of the disease.

PE is the leading cause of maternal and neonatal morbidity and mortality and affects $2-8 \%$ of pregnancies worldwide, with a higher prevalence in developing countries. ${ }^{34}$ The aetiology of PE remains largely unknown, but immunological and angiogenic factors are known to play a role in the development of the disease. ${ }^{5}$ Placentation in early pregnancy appears to involve specific maternal immune responses to fetal alloantigens. $^{7}$ In addition, an imbalance of proangiogenic and antiangiogenic factors has been demonstrated, specifically increased levels of the antiangiogenic soluble fms-like tyrosine kinase 1 , and reduced levels of proangiogenic placental growth factor. ${ }^{8}$ These changes appear in the circulation before clinical signs are apparent; thus, measuring circulating angiogenic proteins may serve as a diagnostic and screening tool for PE.

Epidemiological studies over the past five decades have provided robust and consistent support for the clinical observation that $\mathrm{PE}$ 
runs in families. ${ }^{9-11}$ Deciphering the genetic involvement in PE is challenging, not least because the phenotype is expressed only in pregnant women. ${ }^{12}$ Moreover, it is necessary to consider two genotypes, that of the mother and that of the fetus which includes genes inherited from the mother and father. After almost two decades of molecular research, no genetic variants have been reproducibly associated with $\mathrm{PE}$. Genome-wide linkage studies of PE have revealed a number of maternal susceptibility loci; however, they have not been independently replicated. ${ }^{13}$ Moreover, a large number of candidate genes have been tested addressing mainly the maternal genotype and the susceptibility loci therein but, in common with the experience in other complex disorders, these have generated inconsistent results. ${ }^{13} 1516$ The identification of genetic variants demands large DNA collections from affected mothers and babies, and also controls with reliable supporting phenotypic data. ${ }^{11}$

PE is also known to have consequences for the longterm health of the mother and her child. In particular, a linkage of the pathophysiology of PE and later cardiometabolic disease has been suggested by large epidemiological studies, but is not well substantiated at a mechanistic level. ${ }^{17}$

The Finnish Genetics of Pre-eclampsia Consortium (FINNPEC) is a cross-sectional case-control cohort collected from the five university hospitals in Finland during 2008-2011. It was established to set up a nationwide clinical and DNA database on women with and without $\mathrm{PE}$, including their partners and infants in order to identify genetic risk factors for PE. The study represents a prospective cohort design with detailed clinical outcome information allowing to characterise accurately the subphenotypes of PE. This study will also provide the opportunity to analyse combined clinical and genetic data. Biobank samples from the first and third trimesters provide a resource for further pregnancy research.

\section{COHORT DESCRIPTION}

Altogether, 1450 patients with PE and 1065 pregnant control women without PE were recruited at the five Finnish university hospitals. There were altogether 1377 full triads (625 PE and 752 control triads), the number of available samples and information is presented in table 1 .

There are two arms of the study. In the prospectively recruited arm, PE women $(n=923)$ and women with normal pregnancies $(n=1009)$ were identified on admission at all university hospitals in Finland (Helsinki, Kuopio, Oulu, Tampere and Turku). The baby and the spouse of each participant (father of the child) were also enrolled for the study (table 1 and figure 1). After the recruitment of a PE woman, a woman with a non-PE pregnancy attending the same clinic was recruited as a control woman. In the retrospectively recruited arm of the study, all women who had been diagnosed with PE or placental insufficiency during their pregnancies were retrospectively identified based on medical records of the hospitals and invited to participate (from years 2000-2008, except in Kuopio University Hospital which recruited from years 1990-2008). These women were first contacted by letter by a physician in the hospital where they were diagnosed and treated for the disorder. Altogether, 583 women participated in the retrospective study, but 56 of them did not fulfil the diagnostic criteria for PE and were included as controls. All participants provided written informed consent.

\section{Inclusion criteria}

Nulliparous or multiparous women with a singleton pregnancy were eligible for the study. PE was defined as hypertension and proteinuria occurring after 20 weeks gestation according to the American College of Obstetricians and Gynecologists (ACOG) 2002 criteria. $^{18}$ Hypertension was defined as systolic blood pressure $\geq 140 \mathrm{~mm} \mathrm{Hg}$ or diastolic blood pressure $\geq 90 \mathrm{~mm} \mathrm{Hg}$. Proteinuria was defined as the urinary excretion of

Table 1 Number of available samples (November 2015)

\begin{tabular}{|c|c|c|c|c|c|c|}
\hline & \multicolumn{3}{|c|}{ Pre-eclampsia $(n=1450)$} & \multicolumn{3}{|c|}{ Control (n=1065) } \\
\hline & Mother & Father & Baby & Mother & Father & Baby \\
\hline Serum 1 Trimester & 267 & & & 282 & & \\
\hline Serum 3 Trimester & 277 & & & 243 & & \\
\hline Antepartum & 150 & & & 42 & & \\
\hline At delivery & 35 & & & 24 & & \\
\hline Postpartum & 92 & & & 177 & & \\
\hline DNA & $\begin{array}{l}P: 879 \\
R: 525\end{array}$ & $P: 677$ & P: 859 & $\begin{array}{l}\mathrm{P}: 922 \\
\mathrm{R}: 55\end{array}$ & 771 & 972 \\
\hline Cord blood & & & 805 & & & 878 \\
\hline Cord plasma & & & 486 & & & 526 \\
\hline Placental tissue, 9-site & 101 & & & 78 & & \\
\hline Background information & $P: 749$ & $P: 616$ & & P: 780 & $P: 701$ & \\
\hline & R: 494 & & & $\mathrm{R}: 52$ & & \\
\hline
\end{tabular}


3264 pregnant women attending one of the five University Hospitals of Finland

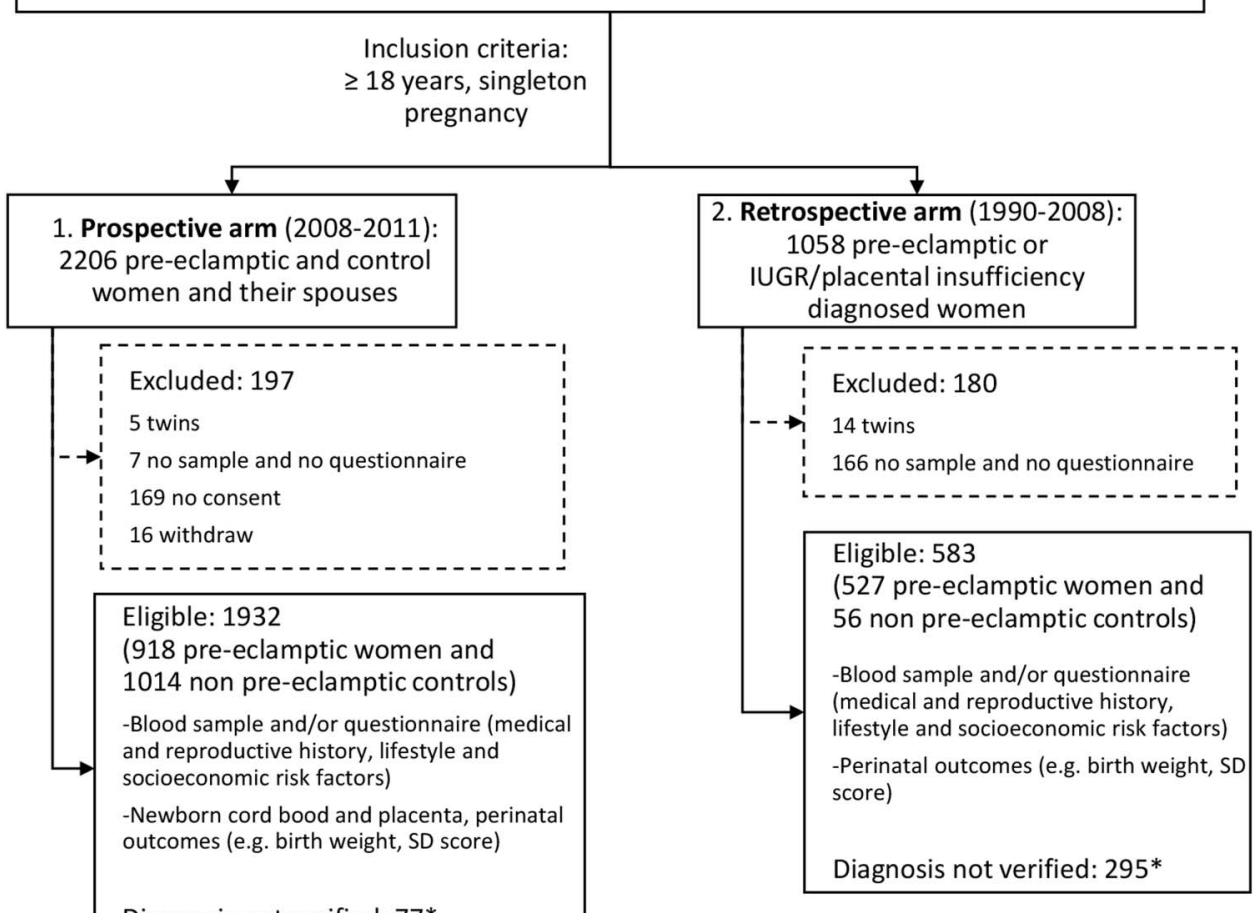

Diagnosis not verified: $77^{*}$

*diagnosis not yet verified by the FINNPEC protocol (November 2015)

Figure 1 Flow chart of the FINNPEC Study. FINNPEC, Finnish Genetics of Pre-eclampsia Consortium. IUGR, intrauterine growth restriction.

$\geq 0.3 \mathrm{~g}$ protein in a 24-hour specimen, or $0.3 \mathrm{~g} / \mathrm{L}$, or two $\geq 1+$ readings on a dipstick in a random urine determination with no evidence of a urinary tract infection. The control group included pregnant women who suffered from proteinuria without hypertension $(n=19)$ and women who suffered from gestational hypertension but did not meet the criteria for PE $(n=111$ and $n=28$ for the prospective and retrospective arms, respectively). Haemolysis, elevated liver enzymes and low platelet count (HELLP) syndrome was diagnosed when at least two of the following criteria were met: lactate dehydrogenase $\geq 235 \mathrm{U} / \mathrm{L}$, alanine aminotransferase $\geq 70 \mathrm{U} / \mathrm{L}$, aspartate aminotransferase $\geq 70 \mathrm{U} / \mathrm{L}$, thrombocytes $\leq 100 \mathrm{E} 9 / \mathrm{L}$. Each diagnosis was ascertained based on hospital records and confirmed independently by a research nurse and a study physician.

\section{Exclusion criteria}

Exclusion criteria were multiple pregnancy, maternal age $<18$ years and the inability to provide an informed consent based on information in Finnish or Swedish.

\section{Background, obstetric and perinatal data}

Extensive information on medical history, family history, obstetric history, pregnancy complications, pregnancy outcome, proteinuria, blood pressure, laboratory measurements, delivery and newborn was obtained from the hospital records ( $\sim 500$ variables).
The participating mother and father were also asked to fill in a detailed questionnaire on background information. The questionnaire included data on prepregnancy weight and height, pregnancy history including previous $\mathrm{PE}$ and family history of $\mathrm{PE}$, personal medical history, family medical history and lifestyle factors (physical activity, smoking and diet). Data on prepregnancy weight, height, diastolic and systolic blood pressure at first antenatal visit, and smoking status were also obtained from primary care maternity records. A woman was considered as a pre-pregnancy smoker if she still smoked 6 months before conception.

PE was defined as early onset when delivery occurred before $34+0$ weeks of gestation and late onset when at $34+0$ weeks of gestation or later. Preterm delivery was defined as delivery occurring before 37 weeks of gestation. Birth weights below -2.0 SD units were classified as small-for-gestational age (SGA) according to Finnish standards. $^{19}$

\section{Socioeconomic factors}

The participating mother and father were asked to fill in a detailed questionnaire on socioeconomic factors including education, occupation and marital status.

\section{Biological samples}

A venous blood sample was drawn from mothers and fathers (table 1). Genomic DNA was extracted from 
whole blood using the NucleoSpin Blood XL DNA extraction kit (Macherey-Nagel $\mathrm{GmbH}$ and Co) or Chemagic Magnetic Separation Module I-machine (Chemagen) and subsequently stored at $-20^{\circ} \mathrm{C}$.

First and third trimester serum samples were collected from a subcohort from the Hospital District of Helsinki and Uusimaa only. First trimester serum samples were obtained via first trimester biochemical screening for fetal chromosome abnormalities (range 9-15 weeks of gestation), and third trimester (range 20-42 weeks of gestation) serum samples were collected at hospitals at recruitment. Table 1 shows the number of antepartum, at delivery and postpartum samples for the third trimester. All samples drawn before contractions were regarded as antepartum samples.

Cord blood, cord plasma and placental samples were collected after delivery from a subcohort. The placental samples were collected according to a nine-site protocol where the placenta is divided into nine sections and a biopsy sample is harvested from each of these sections. The samples were obtained shortly after detachment of the placenta: $70 \%$ of the samples were collected within half an hour, $92.5 \%$ within an hour and the remaining three samples within $80 \mathrm{~min}$ of placental detachment. The samples were subsequently stored in an RNA later solution at $-80^{\circ} \mathrm{C}$.

\section{Basic characteristics}

$\mathrm{PE}$ women were older and suffered more from obesity and pre-existing medical conditions (eg, chronic hypertension, type 1 diabetes and gestational diabetes) compared with control women (table 2). The proportion of primiparous women was also higher in the PE group (table 2). In women who later developed PE, both diastolic and systolic blood pressure were already increased at the first antenatal visit occurring on average at 8.6 \pm 1.5 weeks of gestation. In women suffering from PE, proteinuria occurred on average at $34.7 \pm 3.9$ weeks of gestation. Proteinuria was also observed in some controls but they did not meet the diagnostic criteria for PE.

There were no differences in age, body mass index (BMI) or self-reported chronic hypertension between the fathers in the PE and control groups (table 2).

Altogether, 262 of the 1450 PE pregnancies (18.1\%) were early onset (delivery before $34+0$ weeks of gestation). The women in the early-onset group were older and they had had more pregnancies and deliveries than mothers in the late-onset group (table 3). Furthermore, they had more proteinuria and higher diastolic blood pressure already at the first antenatal visit. They also suffered more frequently from chronic hypertension. There were no differences in smoking before or during pregnancy. These findings support the view that classifying PE into early-onset and late-onset disease differentiates two distinct clinical forms with pathophysiologically specific features.

Women with PE more often reported a history of PE (14.8\% in women with $\mathrm{PE}$ vs $1.7 \%$ in controls). These data were based on hospital records and/or questionnaires. The possibility for recall bias must be acknowledged. More detailed information will be published in the near future after information on PE history has been verified solely from the hospital records.

In the FINNPEC Study, controls were more often smokers before and during pregnancy than women with $\mathrm{PE}$. We also detected a trend for greater proportion of SGA babies for those women with PE who smoked during pregnancy (data not shown).

\section{FINDINGS UNTIL NOW}

The established cohort holds both clinical and genetic information of mother-infant-father triads representing a resource for studying the pathogenesis of the disease as well as later implications in the mother, father and the offspring. Furthermore, placental samples collected will provide an important addition for future research.

The research so far from the FINNPEC has encompassed studies on candidate genes (heme oxygenase-1 promoter and CYP subfamily 2J polypeptide 2), ${ }^{20}{ }^{21}$ Sanger and next-generation sequencing ${ }^{22}$ and various studies on the placenta. ${ }^{24}{ }^{25}$ FINNPEC has also served as a replication cohort in various collaborative efforts to identify genetic risk factors for PE. ${ }^{15} 26$

Ongoing studies focus on elucidating the role of immunogenetic and metabolic factors in PE. Metabolomic studies on serum samples collected at the first and third pregnancy trimesters, respectively, and on fetal cord plasma will be performed in collaboration with researchers from the University of Eastern Finland.

\section{Family study}

The Finnish Pre-eclampsia Family Study ${ }^{14} 27$ will be expanded by families with $\mathrm{PE}$ recruited from the FINNPEC cohort. Those participants who have reported in the background information questionnaire having first-degree relatives with a history of $\mathrm{PE}$ will be requested to contact their relatives and provide them with oral or written information about the FINNPEC Study and the research group's contact information with the support of an information letter. First-degree and second-degree relatives (parents, siblings, grandparents, uncles, aunts and cousins) of the study participants will be recruited. The protocol for collecting the families with $\mathrm{PE}$ has already been approved by the Ethics Committee.

\section{Long-term follow-up}

$\mathrm{PE}$ is known to have consequences for the long-term health of the mother and child. ${ }^{17}$ There are very little long-term data on the morbidity and mortality of fathers. Thus, data on morbidity and mortality (focusing on cardiometabolic disease) will be collected from mothers and fathers through links to the nationwide health registers including the Hospital Discharge Register with data on hospital inpatient and outpatient 
Table 2 Maternal, perinatal and paternal characteristics of pre-eclamptic and control groups in FINNPEC

\begin{tabular}{|c|c|c|c|c|}
\hline Maternal or perinatal characteristics & $\begin{array}{l}\text { Pre-eclampsia } \\
(n=1450)\end{array}$ & Control $(n=1065)$ & $\begin{array}{l}\text { p } \\
\text { Value }^{*}\end{array}$ & p Value \\
\hline Age at delivery, year & $30.3 \pm 5.5(1449)$ & $29.8 \pm 5.2$ & 0.015 & \\
\hline Gravity & $1.9 \pm 1.6$ & $2.3 \pm 1.7$ & $<0.001$ & \\
\hline Parity & $0.5 \pm 1.1$ & $0.8 \pm 1.3$ & $<0.001$ & \\
\hline Primiparous & $1065(73.5 \%)$ & $596(56.0 \%)$ & $<0.001$ & \\
\hline Weight, kg (self-reported, pre-pregnancy) & $68.8 \pm 15.1(1447)$ & $66.3 \pm 13.0(1064)$ & $<0.001$ & $<0.001 \ddagger$ \\
\hline Height, $\mathrm{m}$ & $1.65 \pm 0.06(1449)$ & $1.66 \pm 0.06(1064)$ & 0.001 & \\
\hline BMI, $\mathrm{kg} / \mathrm{m}^{2}$ (self-reported, pre-pregnancy)§ & $25.3 \pm 5.2(1447)$ & $24.1 \pm 4.4(1063)$ & $<0.001$ & $<0.001 \ddagger$ \\
\hline Systolic blood pressure at first antenatal visit, $\mathrm{mm} \mathrm{Hg}$ & $125 \pm 13(1407)$ & $119 \pm 13(1023)$ & $<0.001$ & $<0.001 \rrbracket$ \\
\hline Diastolic blood pressure at first antenatal visit, $\mathrm{mm} \mathrm{Hg}$ & $78 \pm 10(1407)$ & $73 \pm 10(1023)$ & $<0.001$ & $<0.0019$ \\
\hline Highest systolic blood pressure, $\mathrm{mm} \mathrm{Hg}^{\star \star}$ & $167 \pm 19$ & $131 \pm 17(1063)$ & $<0.001$ & $<0.001 \rrbracket$ \\
\hline Highest diastolic blood pressure, $\mathrm{mm} \mathrm{Hg}$ & $110 \pm 9$ & $87 \pm 11(1063)$ & $<0.001$ & $<0.0019$ \\
\hline Proteinuria (maximum), g/24 hour & $4.2 \pm 4.1(1283)$ & & - & \\
\hline Smoking before pregnancy & $313(22.2 \%)(1396)$ & $\begin{array}{l}288(27.4 \%) \\
(1037)\end{array}$ & 0.003 & \\
\hline Smoking during pregnancy & $108(7.6 \%)(1404)$ & $\begin{array}{l}121(11.5 \%) \\
(1052)\end{array}$ & 0.005 & \\
\hline Chronic hypertension†† & $259(17.9 \%)$ & $65(6.1 \%)$ & $<0.001$ & \\
\hline Gestational hypertensionł‡ & - & $139(13.1 \%)$ & - & \\
\hline Gestational diabetes mellitus & $207(14.3 \%)$ & $89(8.4 \%)$ & $<0.001$ & \\
\hline Pregestational diabetes mellitus & $47(3.2 \%)$ & $9(0.8 \%)$ & $<0.001$ & \\
\hline Type 1 diabetes & $38(2.6 \%)$ & $6(0.6 \%)$ & $<0.001$ & \\
\hline Type 2 diabetes & $9(0.6 \%)$ & $3(0.3 \%)$ & 0.223 & \\
\hline \multicolumn{5}{|l|}{ Mode of delivery } \\
\hline Vaginal & $757(52.3 \%)$ & 889 (83.5\%) & & \\
\hline Caesarean section & $693(47.8 \%)$ & $175(16.4 \%)$ & & \\
\hline Birth weight, g & $2692 \pm 897$ & $3455 \pm 687$ & $<0.001$ & $<0.001 \S \S$ \\
\hline Relative birth weight, SD & $-1.0 \pm 1.3(1449)$ & $-0.2 \pm 1.1$ & $<0.001$ & $<0.001 \S \S$ \\
\hline SGA & $306(21.1 \%)$ & $71(6.7 \%)$ & $<0.001$ & \\
\hline Gestational weeks & $36.4 \pm 3.5$ & $39.3 \pm 2.3$ & $<0.001$ & $<0.001 \ddagger$ \\
\hline \multicolumn{5}{|l|}{ Paternal characteristics } \\
\hline Age, years & $32.5 \pm 6.3(699)$ & $32.3 \pm 5.9(854)$ & 0.478 & \\
\hline BMI, $\mathrm{kg} / \mathrm{m}^{2}$ (self-reported) & $26.2 \pm 3.9(548)$ & $26.1 \pm 3.5(611)$ & 0.568 & \\
\hline $\begin{array}{l}\text { Chronic hypertension (requiring medication, } \\
\text { self-reported) }\end{array}$ & $14(2.6 \%)(545)$ & $9(1.5 \%)(616)$ & 0.176 & \\
\hline
\end{tabular}

()Number of available information/samples unless from all.

Data are presented as mean $\pm S D$ or percentages.

Bold text shows $p$ values $<0.05$.

*Unadjusted.

†Adjusted.

$\ddagger$ Adjusted for parity, mother's age at birth.

$\S B$ Based on weight and height before pregnancy, self-reported at first antenatal visit.

ПAdjusted for parity, mother's age at birth, pre-pregnancy BMI.

${ }^{*}$ When highest diastolic value recorded.

††Systolic blood pressure $\geq 140 \mathrm{~mm} \mathrm{Hg}$ and/or diastolic blood pressure $\geq 90 \mathrm{~mm} \mathrm{Hg}$ detected before 20 weeks of gestation.

$\ddagger \ddagger$ Blood pressure $\geq 140 / 90$, no proteinuria.

$\S \S$ Adjusted for parity, mother's age at birth, gestation weeks, pre-pregnancy BMI, chronic hypertension, gestational diabetes, pregestational diabetes mellitus.

BMI, body mass index; FINNPEC, Finnish Genetics of Pre-eclampsia Consortium; SGA, small-for-gestational age.

care and Cause-of-Death Register in collaboration with the National Institute for Health and Welfare (THL) and Statistics Finland. Furthermore, data on familial PE history data will be collected through linkage to these registers. Linkage to health registers is possible through the Finnish personal identification numbers.

\section{International collaboration}

FINNPEC has participated in the genetic studies of PE in Central Asian and European populations
(InterPregGen), which was the largest investigation on the maternal and fetal genetic factors underlying PE until now (http://www.interpreggen.org). ${ }^{28}$ It was funded by the European Community's Seventh Framework Programme. Six hundred PE women, 600 affected fetuses and 600 healthy pregnant control women from the FINNPEC cohort were included in the follow-up genotyping after the genome-wide association screening was performed in the discovery cohorts. FINNPEC also participates in the Global Pregnancy 
Table 3 Early-onset (delivery before $34+0$ weeks of gestation) versus late-onset pre-eclampsia

\begin{tabular}{|c|c|c|c|c|c|c|}
\hline \multirow{2}{*}{$\begin{array}{l}\text { Maternal or perinatal characteristics } \\
\text { Age, year }\end{array}$} & \multicolumn{2}{|c|}{$\begin{array}{l}\text { Pre-eclampsia } \\
\text { Early vs late onset }(n=262) \\
(n=1188)\end{array}$} & \multirow{2}{*}{$\begin{array}{l}\begin{array}{l}\text { Control } \\
(n=1065)\end{array} \\
29.8 \pm 5.2\end{array}$} & \multirow{2}{*}{$\begin{array}{l}\text { p Value } \\
\text { early vs late } \\
<0.001\end{array}$} & \multirow{2}{*}{$\begin{array}{l}\text { p Value } \\
\text { early vs control } \\
<0.001\end{array}$} & \multirow{2}{*}{$\begin{array}{l}\begin{array}{l}\text { p Value } \\
\text { late vs } \\
\text { control }\end{array} \\
0.239\end{array}$} \\
\hline & $31.5 \pm 6.1(261)$ & $30.0 \pm 5.3$ & & & & \\
\hline Gravity & $2.3 \pm 2.0$ & $1.9 \pm 1.5$ & $2.3 \pm 1.7$ & 0.014 & 0.934 & $<0.001$ \\
\hline Primiparous & $176(67.2 \%)$ & $889(74.8 \%)(1187)$ & $596(56.0 \%)$ & 0.013 & 0.001 & $<0.001$ \\
\hline Weight, kg (self-reported, pre-pregnancy) & $68.9 \pm 14.6(261)$ & $68.8 \pm 15.2(1186)$ & $66.3 \pm 13.0(1064)$ & $0.713^{*}$ & $0.006^{\star}$ & $<0.001^{*}$ \\
\hline BMI, kg/m² (self-reported, pre-pregnancy) & $25.6 \pm 5.1(261)$ & $25.2 \pm 5.3(1186)$ & $24.1 \pm 4.4(1063)$ & $0.542^{*}$ & $<0.001^{*}$ & $<0.001^{*}$ \\
\hline Systolic blood pressure at first antenatal visit, $\mathrm{mm} \mathrm{Hg}$ & $126 \pm 14(256)$ & $124 \pm 13(1151)$ & $119 \pm 13(1023)$ & $0.160 \dagger$ & $<0.001 \dagger$ & $<0.001 \dagger$ \\
\hline Diastolic blood pressure at first antenatal visit, $\mathrm{mm} \mathrm{Hg}$ & $80 \pm 10(256)$ & $77 \pm 10(1151)$ & $73 \pm 10(1023)$ & $0.001 \dagger$ & $<0.001 \dagger$ & $<0.001 \dagger$ \\
\hline Highest systolic blood pressure, $\mathrm{mm} \mathrm{Hg}$ & $177 \pm 19$ & $165 \pm 18$ & $131 \pm 17(1063)$ & $<0.001 \dagger$ & $<0.001 \dagger$ & $<0.001 \dagger$ \\
\hline Highest diastolic blood pressure, $\mathrm{mm} \mathrm{Hg}$ & $115 \pm 10$ & $109 \pm 9$ & $87 \pm 11(1063)$ & $<0.001 \dagger$ & $<0.001 \dagger$ & $<0.001 \dagger$ \\
\hline Proteinuria (maximum), g/24 hours & $6.7 \pm 5.4(254)$ & $3.6 \pm 3.4(1029)$ & & $<0.001 \dagger$ & & \\
\hline Smoking before pregnancy & $43(17.1 \%)(251)$ & $270(23.4 \%)(1145)$ & $288(27.4 \%)(1037)$ & 0.058 & 0.001 & 0.041 \\
\hline Smoking during pregnancy & $26(10.3 \%)(251)$ & $82(7.1 \%)(1153)$ & $121(11.5 \%)(1052)$ & 0.200 & 0.875 & 0.001 \\
\hline Chronic hypertension & $71(27.1 \%)$ & $188(15.8 \%)$ & $65(6.1 \%)$ & $<0.001$ & $<0.001$ & $<0.001$ \\
\hline Gestational diabetes mellitus (diet and/or insulin treated) & $29(11.1 \%)$ & $178(15.0 \%)$ & $89(8.4 \%)$ & 0.096 & 0.173 & $<0.001$ \\
\hline Pregestational diabetes mellitus & $5(1.9 \%)$ & $42(3.5 \%)$ & $9(0.8 \%)$ & 0.175 & 0.133 & $<0.001$ \\
\hline Birth weight, g & $1307 \pm 423$ & $2997 \pm 652$ & $3455 \pm 687$ & $<0.001 \ddagger$ & $<0.001 \ddagger$ & $<0.001 \ddagger$ \\
\hline SGA & $135(51.3 \%)$ & $171(14.4 \%)(1185)$ & $71(6.7 \%)$ & $<0.001$ & $<0.001$ & $<0.001$ \\
\hline Relative birth weight (SD) & $-2.0 \pm 1.0$ & $-0.8 \pm 1.2$ & $-0.2 \pm 1.1$ & $<0.001 \ddagger$ & $<0.001 \ddagger$ & $<0.001 \ddagger$ \\
\hline Gestation weeks & $30.3 \pm 2.4$ & $37.7 \pm 1.9$ & $39.3 \pm 2.3$ & $<0.001^{*}$ & $<0.001^{*}$ & $<0.001^{\star}$ \\
\hline
\end{tabular}

Bold text shows $p$ values $<0.05$

${ }^{*}$ Adjusted for parity, mother's age at birth.

†Adjusted for parity, mother's age at birth, pre-pregnancy BMI.

¥Adjusted for parity, mother’s age at birth, pre-pregnancy BMI, chronic hypertension, gestational diabetes, pregestational diabetes mellitus.

BMI, body mass index; SGA, small-for-gestational age. 
Collaboration (CoLab), a major Bill and Melinda Gates Foundation supported initiative focused on adverse pregnancy outcomes and achieving a better understanding of their causes. ${ }^{29}$ CoLab is the largest international initiative until now to understand major pregnancy disorders in a global setting.

\section{STRENGTHS AND LIMITATIONS}

A major strength of this study is its large multicentre prospective design with opportunities for follow-ups as well as the recalling of participants for future studies. The established cohort holds both clinical and genetic information of mother-infant-father triads representing a resource for studying the pathogenesis of PE. Furthermore, maternal biological samples (first and third trimester serum and placenta) will provide additional information for PE research.

In addition, one of the strengths of the current study is the ascertainment of the outcome: each diagnosis was ascertained based on hospital records and confirmed independently by a research nurse and a study physician. When rediagnosed carefully using this protocol, not all recruited cases meet the criteria for PE. Most of these women suffered from gestational hypertension without proteinuria.

The majority of genetic studies of PE have until now focused on maternal genetic contribution. However, owing to the placental origin of $\mathrm{PE}$, it is likely that fetal and/or paternal genetic factors play a central role in the pathogenesis. Evidence supports the idea that paternal and/or fetal genetic factors might contribute to the risk of PE. ${ }^{30}{ }^{31} \mathrm{It}$ has been shown that men born to a PE pregnancy are twice as likely to father a PE pregnancy. ${ }^{30}$ In addition, men who have fathered a PE pregnancy are nearly twice as likely to father a PE pregnancy with a different woman. ${ }^{31}$ Thus, it is important to include the father and the offspring in genetic studies of PE. The study design at FINNPEC opens up this possibility for detecting maternal, paternal and fetal genes and their interactions.

Association between PE and the development of maternal cardiovascular diseases (CVDs) later in life has been well established through epidemiological research. ${ }^{17}$ Follow-up studies that will be carried out in FINNPEC through linkage of the national data sources may help identify prognostic subgroups of mothers, fathers and infants that have a linkage between PE and later life CVD and other non-communicable diseases. In future, this may provide tools for early screening and targeted postpartum preventive strategies.

The FINNPEC cohort also includes information on socioeconomic position such as educational attainment and occupational status, which are currently being analysed. This is important because the role of socioeconomic position as a risk factor of $\mathrm{PE}$ has been little studied.

It must be acknowledged that a large unselected material would have had more epidemiological advantages. This cohort is not directly generalisable to the general population since the participants represented a Finnish population of women with PE who were referred to university hospitals and thus may represent a more severe end of the disease. However, apart from serving as a tertiary centre of a larger catchment area, all these hospitals serve as primary delivery hospitals for a smaller catchment area. The incidence of early-onset disease (18.1\%) was comparable to reports in population-based studies, ${ }^{32}$ whereas the incidence of HELLP $(7.4 \%)$ was quite high. However, the most severe cases are missing as deliveries had taken place immediately before recruitment could take place.

\section{Author affiliations}

${ }^{1}$ Medical and Clinical Genetics, University of Helsinki and Helsinki University Hospital, Helsinki, Finland

${ }^{2}$ Obstetrics and Gynecology, University of Helsinki and Helsinki University

Hospital, Helsinki Finland

${ }^{3}$ Chronic Disease Prevention Unit, National Institute for Health and Welfare, Helsinki, Finland

${ }^{4}$ Children's Hospital, Helsinki University Hospital and University of Helsinki, Helsinki, Finland

${ }^{5}$ PEDEGO Research Unit, MRC Oulu, Oulu University Hospital and University of Oulu, Oulu, Finland

${ }^{6}$ Department of Biosciences and Nutrition, and Science for Life Laboratory, Karolinska Institutet, Stockholm, Sweden

${ }^{7}$ Molecular Neurology Research Program, University of Helsinki, Helsinki,

Finland

${ }^{8}$ Folkhälsan Institute of Genetics, Helsinki, Finland

${ }^{9}$ Division of Cardiovascular Medicine, University of Cambridge, Cambridge, UK

${ }^{10}$ Department of Government Services, National Institute for Health and Welfare, Helsinki, Finland

${ }^{11}$ Institute for Molecular Medicine Finland, University of Helsinki, Helsinki, Finland

Acknowledgements The authors are indebted to all the FINNPEC study participants. They appreciate the contribution of the present or former members of the FINNPEC Study Group: Tia Aalto-Viljakainen, Jenni HeikkinenEloranta, Reija Hietala, Miira Klemetti, Susanna Sainio, Terhi Saisto and Sanna Suomalainen-König (Helsinki University Hospital), Eeva Ekholm and Kaarin Mäkikallio-Anttila (Turku University Central Hospital), Marja Vääräsmäki (Oulu University Hospital), Leena Georgiadis and Leea Keski-Nisula (Kuopio University Hospital), Jukka Uotila (Tampere University Hospital), Sanna Heino, Tea Kaartokallio, Inkeri Lokki ja Marja Vilkki (University of Helsinki). The expert technical assistance of Eija Kortelainen, Susanna Mehtälä, Hanna Nurmi, Aija Lähdesmäki, Satu Leminen, and Christina Salmén is gratefully acknowledged. The authors also thank M.Soc.Sc. Taina Rönkkö for the language review.

Collaborators Collaboration is possible through specific research projects Potential collaborators are invited to contact the primary investigator $\mathrm{HL}$ : hannele.laivuori@helsinki.fi.

Contributors SH, EK, JK, KK, AP and HL were involved in design. The FINNPEC Study Group was involved in collection of data. TJ and HL were involved in data analysis and interpretation. TJ, SH, EK, JK, KK, AP and HL were involved in manuscript writing and revision. All authors read and approved the final manuscript.

Funding The study was supported by Jane and Aatos Erkko Foundation, Päivikki and Sakari Sohlberg Foundation, Academy of Finland (grants 121196, 134957, and 278941), Research Funds of the University of Helsinki, Government special state subsidy for health sciences (In Finnish; Erityisvaltionosuus) at the Hospital District of Helsinki and Uusimaa, The Finnish Medical Foundation, Finska Läkaresällskapet, Novo Nordisk Foundation, Finnish Foundation for Pediatric Research, Emil Aaltonen Foundation, and Sigrid Jusélius Foundation. 
Competing interests None declared.

Ethics approval The FINNPEC Study protocol was approved by the coordinating Ethics Committee of the Hospital District of Helsinki and Uusimaa (149/E0/2007).

Provenance and peer review Not commissioned; externally peer reviewed.

Data sharing statement The authors confirm that some access restrictions apply to the data. The researchers interested in using the data must obtain approval from the FINNPEC Board (steering committee). The researchers using the data are required to follow the terms of a number of clauses designed to ensure the protection of privacy and compliance with relevant Finnish laws. Data requests may be subject to further review by the Ethics Committee and may also be subject to individual participant consent.

Open Access This is an Open Access article distributed in accordance with the Creative Commons Attribution Non Commercial (CC BY-NC 4.0) license, which permits others to distribute, remix, adapt, build upon this work noncommercially, and license their derivative works on different terms, provided the original work is properly cited and the use is non-commercial. See: http:// creativecommons.org/licenses/by-nc/4.0/

\section{REFERENCES}

1. Sibai B, Dekker G, Kupferminc M. Pre-eclampsia. Lancet 2005;365:785-99.

2. Walker JJ. Pre-eclampsia. Lancet 2000;356:1260-5.

3. Steegers EA, von Dadelszen P, Duvekot JJ, et al. Pre-eclampsia. Lancet 2010;376:631-44.

4. Khan KS, Wojdyla D, Say L, et al. WHO analysis of causes of maternal death: a systematic review. Lancet 2006;367:1066-74.

5. Ahn H, Park J, Gilman-Sachs A, et al. Immunologic characteristics of preeclampsia, a comprehensive review. Am J Reprod Immunol 2011:65:377-94.

6. Levine RJ, Maynard SE, Qian C, et al. Circulating angiogenic factors and the risk of preeclampsia. N Engl J Med 2004;350:672-83.

7. Redman CW, Sargent IL. Immunology of pre-eclampsia. Am $J$ Reprod Immunol 2010;63:534-43.

8. Maynard SE, Karumanchi SA. Angiogenic factors and preeclampsia. Semin Nephrol 2011;31:33-46.

9. Chesley LC, Annitto JE, Cosgrove RA. The familial factor in toxemia of pregnancy. Obstet Gynecol 1968;32:303-11.

10. Arngrimsson R, Björnsson S, Geirsson RT, et al. Genetic and familial predisposition to eclampsia and pre-eclampsia in a defined population. Br J Obstet Gynaecol 1990;97:762-9.

11. Morgan L. Update on genetics of pre-eclampsia. Pregnancy Hypertens 2013;3:59-60.

12. Williams PJ, Broughton Pipkin F. The genetics of pre-eclampsia and other hypertensive disorders of pregnancy. Best Pract Res Clin Obstet Gynaecol 2011;25:405-17.

13. Moses EK, Lade JA, Guo G, et al. A genome scan in families from Australia and New Zealand confirms the presence of a maternal susceptibility locus for pre-eclampsia, on chromosome 2. Am J Hum Genet 2000;67:1581-5

14. Laivuori $\mathrm{H}$, Lahermo $\mathrm{P}$, Ollikainen $\mathrm{V}$, et al. Susceptibility loci for preeclampsia on chromosomes 2p25 and 9p13 in Finnish families. Am J Hum Genet 2003;72:168-77.
15. Johnson MP, Brennecke SP, East CE, et al. Genome-wide association scan identifies a risk locus for preeclampsia on 2q14, near the inhibin, beta B gene. PLOS ONE 2012;7:e33666.

16. Chappell S, Morgan L. Searching for genetic clues to the causes of pre-eclampsia. Clin Sci (Lond) 2006;110:443-58.

17. Brown MC, Best KE, Pearce MS, et al. Cardiovascular disease risk in women with pre-eclampsia: systematic review and meta-analysis. Eur J Epidemiol 2013;28:1-19.

18. ACOG Committee on Practice Bulletins-Obstetrics. ACOG practice bulletin. Diagnosis and management of preeclampsia and eclampsia. Number 33, January 2002. Obstet Gynecol 2002;99:159-67.

19. Pihkala J, Hakala T, Voutilainen $P$, et al. [Characteristic of recent fetal growth curves in Finland]. Duodecim 1989;105:1540-6.

20. Herse F, Lamarca B, Hubel CA, et al. Cytochrome P450 subfamily $2 \mathrm{~J}$ polypeptide 2 expression and circulating epoxyeicosatrienoic metabolites in preeclampsia. Circulation 2012;126:2990-9.

21. Kaartokallio T, Klemetti MM, Timonen A, et al. Microsatellite polymorphism in the heme oxygenase-1 promoter is associated with nonsevere and late-onset preeclampsia. Hypertension 2014;64:172-7.

22. Lokki Al, Aalto-Viljakainen T, Meri S, et al. Genetic analysis of membrane cofactor protein (CD46) of the complement system in women with and without preeclamptic pregnancies. PLOS ONE 2015;10:e0117840.

23. Kaartokallio T, Cervera A, Kyllönen A, et al. Gene expression profiling of pre-eclamptic placentae by RNA sequencing. Sci Rep 2015;5:14107.

24. Lokki Al, Heikkinen-Eloranta J, Jarva $\mathrm{H}$, et al. Complement activation and regulation in preeclamptic placenta. Front Immunol 2014;5:312.

25. Reichhardt MP, Jarva $\mathrm{H}$, Lokki Al, et al. The salivary scavenger and agglutinin (SALSA) in healthy and complicated pregnancy. PLOS ONE 2016;11:e0147867.

26. Johnson MP, Brennecke SP, East CE, et al. Genetic dissection of the pre-eclampsia susceptibility locus on chromosome $2 q 22$ reveals shared novel risk factors for cardiovascular disease. Mol Hum Reprod 2013;19:423-37.

27. Majander KK, Villa PM, Kivinen K, et al. A follow-up linkage study of Finnish pre-eclampsia families identifies a new fetal susceptibility locus on chromosome 18. Eur J Hum Genet 2013;21:1024-6.

28. Morgan L, McGinnis R, Steinthorsdottir V, et al. InterPregGen: genetic studies of pre-eclampsia in three continents. Nor Epidemiol 2014;:24:141-6.

29. Roberts JM, Mascalzoni D, Ness RB, et al., Global Pregnancy Collaboration. Collaboration to understand complex diseases: preeclampsia and adverse pregnancy outcomes. Hypertension 2016;67:681-7.

30. Esplin MS, Fausett MB, Fraser A, et al. Paternal and maternal components of the predisposition to preeclampsia. N Engl J Med 2001;344:867-72.

31. Lie RT, Rasmussen $\mathrm{S}$, Brunborg $\mathrm{H}$, et al. Fetal and maternal contributions to risk of pre-eclampsia: population based study. BMJ 1998;316:1343-7.

32. Kenny LC, Black MA, Poston L, et al. Early pregnancy prediction of preeclampsia in nulliparous women, combining clinical risk and biomarkers: the Screening for Pregnancy Endpoints (SCOPE) international cohort study. Hypertension 2014;64:644-52. 